\title{
Accueillir, vêtir, nourrir, instruire, éduquer et soigner : la protection de l'enfance à l'Hospice Saint-Joseph des Soeurs Grises de Montréal (1854-1911)
}

\section{Marie-Christine Giroux}

Volume 65, numéro 2-3, automne 2011, hiver 2012

URI : https://id.erudit.org/iderudit/1018242ar

DOI : https://doi.org/10.7202/1018242ar

Aller au sommaire du numéro

Éditeur(s)

Institut d'histoire de l’Amérique française

ISSN

0035-2357 (imprimé)

1492-1383 (numérique)

Découvrir la revue

Citer cet article

Giroux, M.-C. (2011). Accueillir, vêtir, nourrir, instruire, éduquer et soigner : la protection de l'enfance à l'Hospice Saint-Joseph des Soeurs Grises de Montréal (1854-1911). Revue d'histoire de l'Amérique française, 65(2-3), 153-178.

https://doi.org/10.7202/1018242ar
Résumé de l'article

Dans la seconde moitié du XIX ${ }^{\mathrm{e}}$ siècle à Montréal, la bourgeoisie montante s'intéresse de plus en plus à la misère quotidienne urbaine. Avec l'appui de l'élite cléricale, elle fonde quelques institutions destinées à certaines clientèles, comme les enfants victimes de l'industrialisation et de l'urbanisation. C'est dans ce contexte qu'Olivier Berthelet, bourgeois canadien-français, fonde l'Hospice Saint-Joseph de Montréal. En 1854, cette institution charitable passe aux mains des Soeurs Grises qui y accueillent principalement des " orphelines ». Dès lors, les religieuses cherchent à s'adapter aux besoins matériels, éducatifs et spirituels des familles, et ce, en composant avec les objectifs de régulation sociale à l'origine de l'institution. Dans le cadre de la société patriarcale, elles parviennent à administrer l'Hospice bien qu'elles se heurtent constamment à des problèmes de sous-financement et de manque de personnel. Ces difficultés récurrentes les mèneront graduellement à transformer la vocation charitable de l'institution jusqu'à ce qu'elle devienne l'École ménagère Saint-Joseph en 1911. À plusieurs égards, ce cas d'étude intéressant illustre bien les limites du réseau d'assistance catholique mis en place au milieu du XIX ${ }^{\mathrm{e}}$ siècle.
Tous droits réservés (C Institut d’histoire de l’Amérique française, 2013
Ce document est protégé par la loi sur le droit d'auteur. L'utilisation des services d'Érudit (y compris la reproduction) est assujettie à sa politique d'utilisation que vous pouvez consulter en ligne. 


\title{
Accueillir, vêtir, nourrir, instruire, éduquer et soigner : la protection de l'enfance à l'Hospice Saint- Joseph des Sours Grises de Montréal (I854-I9II)
}

\author{
MARIE-Christine Giroux \\ Département d'histoire \\ Université du Québec à Montréal
}

\begin{abstract}
RÉSumÉ - Dans la seconde moitié du XIXe siècle à Montréal, la bourgeoisie montante s'intéresse de plus en plus à la misère quotidienne urbaine. Avec l'appui de l'élite cléricale, elle fonde quelques institutions destinées à certaines clientèles, comme les enfants victimes de l'industrialisation et de l'urbanisation. C'est dans ce contexte qu'Olivier Berthelet, bourgeois canadien-français, fonde l'Hospice Saint-Joseph de Montréal. En 1854, cette institution charitable passe aux mains des Sœurs Grises qui y accueillent principalement des «orphelines ». Dès lors, les religieuses cherchent à s'adapter aux besoins matériels, éducatifs et spirituels des familles, et ce, en composant avec les objectifs de régulation sociale à l'origine de l'institution. Dans le cadre de la société patriarcale, elles parviennent à administrer l'Hospice bien qu'elles se heurtent constamment à des problèmes de sous-financement et de manque de personnel. Ces difficultés récurrentes les mèneront graduellement à transformer la vocation charitable de l'institution jusqu'à ce qu'elle devienne l'École ménagère Saint-Joseph en 1911. À plusieurs égards, ce cas d'étude intéressant illustre bien les limites du réseau d'assistance catholique mis en place au milieu du XIX ${ }^{e}$ siècle.
\end{abstract}

ABSTRACT - In the second half of the nineteenth century in Montreal, the rising bourgeoisieis getting more and more interested in the urban daily misery. With the support of the clerical elite, it founded several institutions for certain type of clients, such as children, victims of industrialization and urbanization. It is in this context that Olivier Berthelet, a French-

1. Cet article reprend sommairement le mémoire de maitrise que nous avons présenté au département d'histoire de l'Université du Québec à Montréal, en juillet 2011, sous le titre Accueillir, vêtir, nourrir, instruire, éduquer et soigner: la protection de l'enfance à l'Hospice Saint-Joseph des Sours Grises de Montréal (1854-1911). Nous tenons à remercier Martin Petitclerc pour ses précieux conseils. 
Canadian middle-class man, founded the Hospice St. Joseph of Montreal. In 1854, this charitable institution passes to the hands of the Grey Nuns who mainly cater to orphans. The Hospice is not intended to discipline the poor families, but rather to adapt to their educational and spiritual materials, family needs. In the context of patriarchal society, the Sisters will arrive to administer their institution for over half a century, constantly hitting problems of underfunding and understaffing. These recurrent problems will lead gradually to transform the charitable purpose of the institution until it closed in 1911 to become Domestic School St. Joseph. In this regard, this interesting case study illustrates the limitations of Catholic support network set up in the middle of the nineteenth century.

$\mathrm{P}$ réoccupé par les bouleversements sociaux apportés par l'urbanisation et l'industrialisation de Montréal, le bourgeois philanthrope Olivier Berthelet, l'un des rares Canadiens français disposant d'une importante fortune $^{2}$, fondait en 1841 un hospice dans le but de venir en aide aux femmes et aux enfants pauvres du faubourg Saint-Antoine. En 1854, il consentait à confier son œuvre à la Communauté des Sœurs de la Charité (Sœurs Grises) de Montréal. Ainsi, comme c'était souvent le cas chez les franco-catholiques, une institution à l'origine laïque passait aux mains des religieuses alors qu'elle commençait à prendre de l'ampleur ${ }^{3}$.

Dans une société libérale où la responsabilité de l'individu constituait le fondement même de l'ordre social, l'État s'impliquait minimalement dans le système d'assistance. Les lois québécoises, différentes des Poor Laws anglaises (à partir de 1601) ou même du Charity Act de l'Ontario (1874), sont demeurées supplétives en matière d'assistance jusqu'en 1921, lorsqu'a été adoptée la Loi sur l'assistance publique ${ }^{4}$. Cette non-intervention de l'État au Québec s'appliquait également au domaine de l'enfance'. Son action se limitait en fait à certaines catégories de personnes comme les malades

2. Huguette Lapointe-Roy, Charité bien ordonnée : le premier réseau de lutte contre la pauvreté à Montréal au $19^{e}$ siècle (Montréal, Boréal, 1987), 114.

3. Johanne Daigle et Dale Gilbert, «Un modèle d'économie sociale mixte : le dynamisme des services sociaux à l'enfance dans la ville de Québec, 1850-1950», Recherches sociographiques, 49,1 (2008): 121.

4. «Cette loi étendra à l'échelle de tout le Québec le principe du financement public de tous les établissements d'assistance, à raison du tiers fourni par la province, d'un autre tiers par la municipalité impliquée, le dernier tiers relevant de la responsabilité de l'institution. » Jean-Marie Fecteau et Janice Harvey, «Le réseau de régulation sociale montréalais», dans Dany Fougères, Normand Perron et al., Histoire de Montréal et de sa région (Québec, Éditions PUL/IQRC, coll. «Les régions du Québec», 2012), $1600 \mathrm{p}$.

5. Éric Vaillancourt, «L’enfant et le réseau charitable catholique montréalais : le cas de la Société de Saint-Vincent-de-Paul, 1848-1930 ", dans Éric Pierre et Jean Trépanier, dir.,Le temps de l'histoire, 5 (septembre 2003): 173 . 
mentaux, les criminels ou les jeunes délinquants ${ }^{6}$. Il laissait par conséquent la responsabilité des populations pauvres à la société civile.

La place laissée vacante par les différentes instances gouvernementales dans le domaine de la charité durant le XIX ${ }^{\mathrm{e}}$ siècle a incité les élites canadiennes-françaises à créer leurs propres services de charité. Elles se sont particulièrement attardées aux enfants négligés des quartiers ouvriers. Cependant, à l'encontre des élites protestantes, les élites canadiennesfrançaises avaient un rôle limité au sein des institutions. Alors que le réseau protestant était coordonné par la bourgeoisie anglophone, le système d'assistance catholique ${ }^{7}$ était dirigé par l'Église ${ }^{8}$. Les problèmes sociaux étaient donc relayés principalement au domaine privé et plus spécifiquement à des groupes confessionnels. L’État reconnaissait ainsi le travail des communautés religieuses à qui il confiait la tâche de secourir les nécessiteux ${ }^{9}$. Par le fait même, l'Église agissait, selon l'historien JeanMarie Fecteau, "comme un corps social auquel on [avait] dévoué un contrôle au moins partiel de segments importants des institutions de régulation sociale ${ }^{10}$ ".

L'hospice, qui s'adressait à une clientèle hétérogène, a été «l'institution caractéristique du XIX ${ }^{e}$ siècle $^{11}$ ». À celui-ci, s'ajoutaient les hôpitaux et les orphelinats qui étaient mis à la disposition des familles. En raison notamment du passage de la société préindustrielle à une société industrielle, la situation de bien des familles était devenue précaire et a fait monter en flèche le nombre d'institutions destinées à l'enfance.

6. Jean-Marie Fecteau, «L'enfance en difficulté au $19^{\mathrm{e}}$ siècle et dans les premières années du $20^{\mathrm{e}}$ siècle : les implications d'un regard sur les cas limites ", dans ibid., 246.

7. Il importe toutefois de préciser que dans ce réseau catholique, deux sous-réseaux agissaient parallèlement, soit un premier, plus massif, pour répondre aux besoins de la population francophone et un second dédié à la population anglophone. Pour en connaître davantage sur cette distinction, consulter Amélie Bourbeau, La réorganisation de l'assistance chez les catholiques montréalais: la Fédération des œuvres de charité canadiennes-françaises et la Federation of Catholic Charities, 1930-1972, thèse de doctorat (histoire), Université du Québec à Montréal, 2009.

8. Il est pertinent de rappeler que d'autres groupes religieux ont également mis sur pied des réseaux d'assistance à Montréal, bien qu'à de plus petites échelles. C'est le cas notamment des Juifs qui se sont regroupés en association afin de secourir les nouveaux arrivants dans le besoin. Pour en savoir plus sur le sujet, consulter Gerald Tulchinsky, "Immigration and Charity in the Montreal Jewish Community Before 1890 », Histoire sociale, 16,32 (novembre 1983): 359-380.

9. Tania Martin, «La mouvance de la culture canadienne-française vue à travers l'évolution d'une institution catholique en région", The Canadian Historical Review, 88,1 (mars 2007): 55.

10. Jean-Marie Fecteau, «La construction d'un espace social: les rapports de l'Église et de l’État et la question de l'assistance publique au Québec dans la seconde moitié du XIX siècle», dans Yvan Lamonde et Gilles Gallichan, dir., L'histoire de la culture et de l'imprimé. Hommages à Claude Galarneau (Québec, Les Presses de l’Université Laval, 1996), 68.

11. Sylvie Côté, «Les orphelinats catholiques au Québec de 1900 à 1945 », Les cahiers de la femme, 7,4 $(1986): 36$. 
C'est dans ce contexte que les Sours de l'Hospice Saint-Joseph ont accueilli, vêtu, nourri, instruit, éduqué et soigné des orphelines, qui allaient constituer leur clientèle principale ${ }^{12}$. Au fil du temps, les Sours ont intégré différents services à l'Hospice Saint-Joseph afin de répondre autant que possible aux besoins des populations pauvres qui se diversifiaient dans leur quartier. Ainsi, en plus des orphelines, des filles illégitimes et des femmes âgées, elles ont offert les services de la Salle d'Asile, du Jardin de l'Enfance, du dispensaire, des visites des pauvres et des malades à domicile et du Dépôt des pauvres ${ }^{13}$.

Entre 1854 et 1911, années qui s'étendent de la prise de possession de l'institution charitable par les Sœurs Grises à sa transformation en une école ménagère, les Sours ont tenté de répondre à leurs visées charitables et éducatives au meilleur des ressources humaines et matérielles dont elles disposaient. Il va sans dire que ces moyens étaient limités dans un contexte où elles devaient prendre soin d'un nombre considérable d'enfants. Inévitablement, entre cette volonté de protection de l'enfance qui les animait et la réalité quotidienne de l'Hospice Saint-Joseph, nous avons trouvé un hiatus fascinant à analyser. La particularité de cette institution par rapport aux autres institutions «d'enfermement» de la seconde moitié du XIX ${ }^{e}$ siècle s'est également révélée intéressante. Nous croyons en effet que cette institution pour enfants n'a pas été, comme le voyaient les élites, un lieu qui servait à préserver du vice les enfants des familles pauvres afin d'assurer le maintien et la reproduction de l'ordre social ${ }^{14}$.

Certes, au-delà de l'amour du prochain qui fondait sa mission charitable, l'Hospice Saint-Joseph était traversé par les rapports sociaux caractéristiques de la société montréalaise de la seconde moitié du XIX ${ }^{\mathrm{e}}$ siècle.

12. D’emblée, il importe de spécifier qu'à l'époque, le terme "orphelin» ne se rapporte pas qu'aux enfants dont les deux parents sont décédés, mais plutôt, et même dans la plupart des cas, à des enfants dont un seul parent est décédé ou à des enfants placés dont les deux parents sont toujours vivants. Encore en 1933, les orphelins qui ont toujours au moins un parent constituent une large majorité. Selon les données de la Commission des Assurances sociales de Québec, qui a été la première à relever ces statistiques de façon aussi complète, il a été révélé que dans la province, $21 \%$ des orphelins avaient leurs deux parents vivants, $67 \%$ avaient l'un des deux parents, alors que seulement $12 \%$ étaient des "orphelins complets». Données présentées dans L'Abbé Charles-Édouard Bourgeois, Une richesse à sauver: l'enfant sans soutien (Trois-Rivières, Bien public, 1947), 113.

13. Pour en connaître davantage sur les clientèles desservies et sur les services offerts par les Sœurs de l'Hospice Saint-Joseph, consulter M.-C.Giroux, op. cit.

14. Selon cette logique, explique Jean-Marie Fecteau, dans le contexte de la transition au libéralisme, la famille ouvrière était tenue de plus en plus responsable des problèmes de l'enfance. Ses enfants "victimes» lui étaient par conséquent retirés et systématiquement pris en charge par le biais du placement ou de l'institutionnalisation afin de les "protéger» d'un milieu familial malsain. Jean-Marie Fecteau, $L a$ liberté du pauvre: crime et pauvreté au XIX siècle québécois (Montréal, vlb éditeur, coll. "Études québécoises», 2004). 
En ce sens, les Sœurs qui le géraient étaient confrontées aux valeurs dominantes de genre et de classe de leur époque, qu'elles subissaient et qu'elles tendaient à promouvoir et à reproduire. Toutefois, les sources institutionnelles ${ }^{15}$ nous ont montré que l'Hospice Saint-Joseph ne s'est pas inscrit dans une logique de contrôle social, mais bien dans une vision éducative plus large qui s'adressait à la classe ouvrière et à la classe moyenne. Ainsi, son orphelinat n'aurait pas eu pour but de «briser» les familles en s'immisçant dans leur vie pour lutter contre les problèmes sociaux, mais bien à les soutenir lors d'un moment de crise, voire à offrir à certaines filles une éducation à faibles coûts dans un contexte où le système scolaire était peu développé.

Nous souhaitons dans un premier temps définir l'institution dans ses rapports avec les orphelines, sa composante la plus importante,et leur famille, à travers l'étude de la clientèle, des missions éducative et charitable et du fonctionnement interne de l'Hospice Saint-Joseph. Nous pourrons par la suite observer, à travers les ressources humaines et matérielles de l'institution, que le fort roulement du personnel laïque et religieux et son nombre restreint, combinés à un manque de ressources financières constant, ont fait obstacle au travail des Sours qui administraient l'Hospice sous le regard du Clergé. Nous verrons que ces conditions difficiles ont poussé les administratrices de l'Hospice à lui faire prendre un nouveau virage dans sa vocation au fil de la période à l'étude. Ainsi, nous constaterons que la mission charitable de l'Hospice SaintJoseph a progressivement laissé place à celle de l'enseignement ménager destiné aux jeunes filles de milieux plus aisés. C'est à la fois sur des perspectives de l'historiographie de la régulation sociale, de la famille ouvrière et des femmes dirigeantes des institutions charitables que notre analyse sera fondée.

\section{L'ORPHELINAT DE L'HOSPICE SAINT-JOSEPH}

\section{Portrait de la clientèle}

Plusieurs stratégies se sont imposées pour répondre aux besoins des familles ouvrières fragilisées par l'industrialisation et l'urbanisation. La parenté est toujours demeurée centrale pour les familles qui recherchaient

15. Nous croyons essentiel de mentionner que la majorité de nos sources proviennent d'une numérisation massive qui a été faite par le Centre d'histoire des régulations sociales (CHRS) de l'Université du Québec à Montréal aux archives des Sœurs de la Charité de Montréal. Les registres d'admission de l'Hospice Saint-Joseph ont été les sources principales du mémoire de maîtrise à l'origine du présent article. 
une aide financière ou un refuge temporaire pour leurs enfants. Toutefois, cette solution n'était pas toujours suffisante et les familles devaient recourir à une ou à plusieurs autres stratégies de survie, parmi lesquelles le placement de leur enfant. Les parents, souligne Bettina Bradbury, se tournaient vers les institutions charitables seulement en dernier recours, lorsque le réseau familial ou d'autres types d'arrangements informels n'étaient pas disponibles ${ }^{16}$. Selon elle, les familles de la classe ouvrière usaient donc de la charité pour traverser les temps les plus durs, pendant lesquels les enfants étaient nourris, logés et soignés. Kathy C. Provost a démontré dans son mémoire de maîtrise que le métier du père a clairement influencé la fréquentation scolaire des enfants et leur présence sur le marché du travail ${ }^{17}$. L'état des sources, en ce qui concerne l'Hospice Saint-Joseph, ne nous permet pas d'être catégorique sur ce point. Nous avons tout de même constaté que l'Hospice n'était pas fermé aux enfants de travailleurs non qualifiés, comme les journaliers, peu susceptibles de verser une pension. Ces familles étaient évidemment les plus enclines à connaitre des crises familiales lors d'un cycle économique défavorable ${ }^{18}$.

Nous avons également constaté que plusieurs enfants avaient un père qui exerçait un métier qualifié, voire qui appartenait à la petite-bourgeoisie ou à la classe moyenne. Cela confirme que la pauvreté n’a pas toujours été l'unique raison pour le placement de l'enfant à l'Hospice. En effet, nous avons pu observer que des raisons familiales, et principalement le décès ou la maladie de la mère, ont poussé plusieurs pères à envoyer temporairement leur enfant en institution, de même que des familles plus aisées financièrement ont placé leurs jeunes filles à l'Hospice pour leur offrir une formation ménagère. Pour certains parents de la classe ouvrière, la formation donnée à l'Hospice Saint-Joseph, à l'opposé de celle de la British \& Canadian School étudiée par les historiens Roderick MacLeod et Mary Anne Poutanen ${ }^{19}$, permettait probablement à leurs jeunes filles d'apprendre le métier de domestique. La recherche de meilleures condi-

16. Bettina Bradbury, «The Fragmented Family: Family Strategies in the Face of Death, Illness, and Poverty, Montreal, 1860-1885 ", dans Joy Parr, dir., Childhood and Family in Canadian History (Toronto, McClelland and Stewart, 1982), 109-128.

17. Kathy C. Provost, «Blunted Lives: Working Children in East-End Montreal, 1880-1890», mémoire de maîtrise (sociologie et antropologie), Concordia University, 2006, 78.

18. Marta Danylewycz, «Sexe et classes sociales dans l'enseignement: le cas de Montréal à la fin du $19^{\mathrm{e}}$ siècle», dans Nadia Fahmy-Eid et Micheline Dumont, dir., Maîtresses de maison, maîtresses d'école: femmes, famille et éducation dans l'histoire du Québec (Montréal, Boréal Express, 1983), 99.

19. Roderick Macleod et Mary Anne Poutanen, "Proper Objects of This Institution”: Working Families, Children, and the British \& Canadian School in Nineteenth-Century Montreal", Revue d'histoire de l'éducation, 20,2 (printemps 2008):45. 
tions de vie pour leur enfant, en plus de leur enlever une bouche à nourrir et de leur fournir éventuellement un revenu complémentaire, amenait ainsi certains parents à privilégier le placement en institution ${ }^{20}$. À cet égard, nous croyons que la formation religieuse et ménagère qui était dispensée à l'Hospice Saint-Joseph a pu pousser plusieurs familles à y placer leur fille pour quelques années.

Selon Judith Dulberger, qui critique la théorie de la régulation sociale, l'utilisation de l'institution par les familles ne s'est pas toujours faite par choix désespéré ${ }^{11}$. Selon l'auteure, c'est dans leurs propres intérêts que les parents des familles pauvres permettaient à leur enfant d'aller chercher les soins matériels et éducatifs ainsi qu'une formation en institution. De plus, ces institutions étaient loin d'avoir pour objectif de séparer les enfants de leur famille. Dans le cas de l'Albany Orphan Asylum, sur lequel elle s'est penchée, l'institution visait bien davantage à préserver les familles durant les temps plus difficiles. Ainsi, bien plus que des lieux disciplinaires, les institutions destinées à la protection de l'enfance ont été une alternative positive pour les familles qui en faisaient usage.

Les orphelines qui ont fréquenté l'Hospice Saint-Joseph étaient majoritairement canadiennes-françaises, bien que quelques orphelines immigrantes, principalement d'origine irlandaise, y aient également été admises. C'est avec un bémol, en raison du nombre trop faible de données sur le sujet, que nous affirmons que la plupart d'entre elles provenaient de quartiers ouvriers francophones de Montréal et, dans une plus faible proportion, des régions rurales éloignées. Dans l'ensemble, ces jeunes filles étaient âgées de deux à dix-huit ans. Entre 1854 et 1873, elles ont été en moyenne 107 par année à vivre dans l'institution ${ }^{22}$, alors que pendant la période de 1874 à 1911, 91 en moyenne y ont vécu chaque année.

20. Claudette Lacelle, Les domestiques en milieu urbain canadien au XIXe siècle (Ottawa, lieux et parcs historiques nationaux, Environnement Canada-Parcs, coll. «Études en archéologie, architecture et histoire», 1987), 166.

21. Judith Dulberger, Refuge or Repressor: The Role of the Orphan Asylum in the Lives of Poor Children and Their Families in Late Nineteenth-Century America, thèse de doctorat (histoire), Carnegie Mellon University, 1988.

22. Le nombre annuel d'orphelines est présenté de façon sporadique dans les rapports annuels des Sœurs Grises. Ces rapports nous ont éclairée quant à la présence des orphelines entre les années 1854 et 1876, mais nous avons dû compléter en comptant le nombre d'orphelines qui restaient dans l'institution au 31 décembre de chaque année jusqu'en 1911. Étant donné que les données reliées aux rapports annuels (A.S.G.M. L10 E1 1, Hospice Saint-Joseph de Montréal fondé en l'année 1854. État du personnel, etc., etc. du dit Hospice, 1854-1876) ne sont pas issues de la même source que celles que nous avons comptabilisées à partir des registres (A.S.G.M. F116 11 C2, [Registres d'entrée de l'Hospice Saint-Joseph], non daté, 1-645), nous avons préféré les traiter de façon distincte, séparant ainsi les périodes de 1854 à 1873 et de 1874 à 1911. 
Nombre de facteurs ont contribué à l'augmentation du nombre d'orphelines au cours des années, parmi ceux-ci, notons l’impact des épidémies de variole et de petite vérole, qui ont débuté respectivement en 1885 et 1886, et l'augmentation rapide de la population montréalaise.

L'analyse d'un échantillon de $\operatorname{cas}^{23}$ a révélé que différents types d'orphelines ont fréquenté l'Hospice Saint-Joseph. Nous avons distingué quatre types: les orphelines de père, les orphelines de mère, les "vraies » orphelines dont les deux parents étaient décédés et les orphelines dont les deux parents étaient toujours vivants. Parmi les 221 orphelines de notre échantillon, 51 n'avaient plus de père $(23 \%), 64$ n'avaient plus de mère (29\%), 20 étaient de "vraies orphelines " $(9 \%)$ et les 86 autres avaient toujours leurs deux parents (39\%), des proportions qui sont demeurées somme toute similaires au fil des années.

La durée de leur séjour, de quelques jours, quelques mois ou de quelques années, était souvent influencée par les conditions économiques et familiales du ou des parents. Plusieurs orphelines ont connu des passages de plus courte durée. Notre échantillon nous a permis d'associer ces courts séjours aux enfants qui sont entrés à l'Hospice pour des raisons de crise financière passagère dans leur famille. Le salaire «à bon marché» des journaliers était si faible qu'il ne permettait effectivement aucune flexibilité dans le budget familial ${ }^{24}$. Par conséquent, les parents sans le sou ou sans aide ont parfois cherché quelques mois, voire quelques semaines ou quelques jours, de répit pour leurs enfants chez les Sœurs ${ }^{25}$. Remarquons que 141 des 245 séjours d'orphelines comptabilisés, soit près de $58 \%$ d'entre eux, ont duré moins d'un an. De surcroît, environ le tiers de ces jeunes filles étaient nouvellement admises chaque année, un phénomène de grande mobilité qui, selon l'historienne Huguette Lapointe-Roy, contribuait à alourdir le travail des Sours qui devaient continuellement préparer l'arrivée de l'une et le départ de l'autre ${ }^{26}$.

Nous avons constaté que les Sœurs appréciaient la stabilité dans leur institution et que, par conséquent, elles ont souvent privilégié l'admission des enfants qui pouvaient $y$ vivre à plus long terme. Dans les cas où cette

23. Étant donné l'ampleur des registres d'admission de l'institution, nous avons sélectionné un échantillon de données. Afin de s'assurer du suivi des parcours individuels, cet échantillon a été composé des personnes figurant aux registres dont le nom de famille commençait par la lettre «L", ce qui représentait environ $10 \%$ de l'ensemble de la clientèle de l'institution.

24. Bettina Bradbury, Familles ouvrières à Montréal: âge, genre et survie quotidienne pendant la phase d'industrialisation (Montréal, Boréal, 1995), 106.

25. Ibid.,121.

26. H. Lapointe-Roy, op. cit.,169. 
durée pouvait être fixée dès le début, la personne responsable de l'orpheline convenait avec la Supérieure de la durée d'hébergement sans frais en échange de quoi l'orpheline s'engageait à remplir certaines tâches en fonction de son âge. En août 1899, par exemple, lorsque le père et le frère d'une orpheline récemment sortie suppliaient l'Hospice de la reprendre pour la soustraire au mauvais comportement de sa belle-mère, elle acceptait, mais sous la "condition expresse de signer un papier pour 5 ans ${ }^{27}$ ». Cette stratégie permettait de former les orphelines et, ainsi, de pouvoir compter sur une main-d'œuvre stable qui pourrait servir notamment à l'Ouvroir (la salle de couture), où les compétences de couturières permettaient de rapporter des revenus supplémentaires à l'institution.

Bien qu'on en retrouve peu de traces dans les sources, tout porte à croire que l'institution ait été rapidement dans l'obligation de refuser des enfants puisqu'elle était débordée. Dans cette foulée, les Sœurs ont dû privilégier certains critères de sélection pour discriminer entre toutes celles qui cognaient à leur porte. Il semble qu'on ait privilégié les familles bien intégrées dans le tissu communautaire et, à partir des années 1880, les familles en mesure de verser une pension pour leur fille. La capacité de payer une partie des dépenses d'entretien de l'orpheline a sans doute joué un rôle crucial dans la sélection de la clientèle. Ainsi, plusieurs familles pauvres et mal intégrées dans la communauté se sont vues bloquer l'accès à l'institution. Les sources nous ont révélé qu'à partir du milieu de la décennie 1880, la demande de pensions s'est installée dans l'institution et s'est rapidement répandue pour être exigée à plus de la moitié des orphelines qui y entraient. Parfois s'y ajoutait une exigence relativement à certains besoins de l'enfant. À plusieurs reprises, nous avons constaté, par exemple, que les parents devaient fournir à leur jeune fille des vêtements, des chaussures, des livres et même s'occuper de la laver pendant son séjour. Ce minimum, tout comme les pensions, sauvait des frais et du temps aux Sours et à leur personnel débordé. Cela dit, la vocation religieuse et charitable de l'établissement empêchait visiblement que ces critères soient appliqués trop strictement.

En conformité avec les principes de leur communauté qui mettaient de l'avant une politique d' "open door" pour les enfants non délinquants ${ }^{28}$, les Sours de l'Hospice Saint-Joseph ont accepté de recevoir des enfants

27. A.S.G.M. L10 2H3, L’Hospice Saint-Joseph de Montréal, [copie dactylographiée], Chroniques manuscrites (Octobre 1892 à octobre 1912), 3 août 1899, 191.

28. Dorothy M. Brown et Elizabeth McKeown, The Poor Belong to Us: Catholic Charities and American Welfare (Cambridge, Harvard University Press, 1997), 98. 
qui vivaient dans diverses conditions. Des disparités de clientèles ont ainsi été rencontrées, puisqu'il s'agissait d'une institution qui desservait à la fois des jeunes filles de milieux pauvres ou plus aisés, durant quelques jours, quelques mois ou même quelques années. Ainsi, l'institution s'est montrée ouverte tant aux familles qui recherchaient un refuge temporaire pour leur enfant, le temps que la situation de crise qu'elles vivaient se résorbe, qu'à des familles plus à l'aise qui visaient à leur assurer un avenir meilleur en leur offrant une formation. Dans les deux cas, la routine, la nourriture adéquate et l'hygiène acceptable, accompagnées d'une formation domestique et d'une instruction morale et religieuse, ont motivé plus d'un parent à envoyer leur enfant à l'Hospice Saint-Joseph. Des populations autonomes utilisaient donc stratégiquement des services offerts comme le placement des enfants en institution, à bien d'autres fins que celles de régulation sociale prévues par les élites ${ }^{29}$.

\section{Les missions et le fonctionnement interne}

La rigueur et la régularité permettaient à l'institution de fonctionner malgré les débordements de clientèle. La vie rythmée par les activités religieuses visait, bien évidemment, à faire des jeunes filles de bonnes chrétiennes. L'évaluation fréquente de leurs connaissances religieuses que nous ont révélée les Chroniques $^{30}$ en fait la preuve. La régularité se reflétait dans un horaire ponctué par les repas, la prière et les travaux auquel étaient soumises autant les Sours que les pensionnaires. Le respect de la rigueur de cet horaire était inévitable aux yeux des Sours qui cherchaient à protéger les jeunes filles en leur offrant un mode de vie stable et axé sur la religion.

Les dirigeantes de l'Hospice Saint-Joseph ont dû répondre à des besoins d'assistance tout en se conformant à des exigences de genre et de classe. Elles œuvraient dans un contexte particulier où s'entrecroisaient des normes sociales et des attentes populaires et où elles agissaient avec une part d'autonomie. Ainsi, les Sours devaient à la fois négocier avec des pressions relatives à l'encadrement des nécessiteux et avec une volonté d'aider des personnes en détresse. S'il ne s'agissait pas d'une institution de contrôle social au sens strict, la vie à l'intérieur des murs de l'Hospice était tout de même régulée par certaines valeurs, liées notamment au rôle

29. Louise Bienvenue, «Pierres grises et mauvaise conscience. Essai historiographique sur le rôle de l'Église catholique dans l'assistance au Québec», Études d'histoire religieuse, 69 (2003): 15.

30. Les Chroniques de l'institution étaient rédigées par une Sœur œuvrant dans l’Hospice Saint-Joseph qui racontait au jour le jour les événements entourant, par exemple, les entrées, les sorties, les maladies, les visites reçues et les rapports avec la communauté religieuse en général. 
maternel des femmes et à la hiérarchie sociale. Les Sours Grises de l'Hospice Saint-Joseph devaient donc former les jeunes filles selon les normes et les savoir-faire nécessaires à la maternité et à la domesticité ${ }^{31}$ afin qu'elles deviennent de bonnes épouses et de bonnes mères, mais aussi des femmes assez robustes et travailleuses pour gérer leur ménage ${ }^{32}$.

Avant même la prise de possession de l'Hospice par les Sœurs Grises, les orphelines qui y demeuraient recevaient une instruction "propre à leur condition». Un peu plus tard, les Constitutions de la Communauté des Sœurs Grises confirmaient une telle conception éducative :

7. Les Sœurs donneront à leurs Orphelines l'instruction qui convient à leur âge et à leur condition. Elles les instruiront selon la méthode utilisée dans l’Institut, et elles s'efforceront d'exciter parmi elles une sage émulation.

8. Une partie essentielle de l'éducation qu'elles leur doivent est de leur apprendre les ouvrages propres à leur sexe, et de les exercer dans les soins du ménage, selon que les enfants en sont capables. Elles formeront aussi à la politesse selon leur âge et leur condition ${ }^{33}$.

Aux yeux des élites, l'éducation offerte dans les institutions religieuses apparaissait comme un instrument privilégié pour maintenir l'intégrité des frontières sociales. Ainsi, les membres des "classes inférieures» devaient accepter de se conformer aux exigences de leur destinée sociale et l'accepter comme une volonté providentielle. Dans cette optique, l'éducation qui était donnée aux orphelines ne devait pas viser leur mobilité sociale, mais devait plutôt respecter scrupuleusement les attentes sociales à l'égard de la classe ouvrière. Les Sours devaient ainsi rendre un enseignement conforme au milieu social de leurs orphelines, en évitant une instruction trop avancée comme celle proposée dans les pensionnats pour les jeunes filles bourgeoises ${ }^{34}$.

Les Sœurs devaient, comme Sylvie Côté l'a également constaté dans son étude sur l'Hospice du Sacré-Cœur de Sherbrooke, inculquer très tôt aux orphelines le goût du travail, valeur jugée essentielle ${ }^{35}$. Les jeunes

31. Denise Lemieux, "La socialisation des filles dans la famille», dans M. Dumont et N. Fahmy-Eid, dir., op. cit., 240.

32. B. Bradbury, Familles ouvrières..., op. cit., 201.

33. BAnQ, MIC B524 13837 GEN, Constitutions des Seurs de la Charité, administratrices de l'HôpitalGénéral de Montréal dites vulgairement Scuurs Grises (Montréal, Imprimerie de l'Hôpital Général, non daté), 310-335.

34. Marie-Paule Malouin, Ma seur, à quelle école alliez-vous? Deux écoles de filles à la fin du XIXe siècle (Montréal, Fides, 1985), 146.

35. Sylvie Côté, "L'œuvre des orphelins à l'Hospice du Sacré-Cœur de Sherbrooke (1875-1965)», Journal of Eastern Townshpis Studies, 16 (printemps 2000): 6 [En ligne] consultée le 25 juillet 2010. 
filles étaient par conséquent rapidement mises au travail, selon les capacités liées à leur âge. En plus de leur permettre de contribuer aux frais de l'institution, leur participation aux travaux des Sours les formait à devenir de bonnes "maîtresses de maison ${ }^{36}$ ». À l'Hospice Saint-Joseph, l'Ouvroir leur apprenait la couture et les mettait «en état de gagner leur vie ${ }^{37}$ ». Très tôt, les fillettes devaient donc apprendre à coudre et à tricoter ${ }^{38}$.

La valorisation du travail (de couture ou autre), tout comme l'historienne Pascale Quincy-Lefebvre l'a démontré pour les institutions françaises, devait assurer la survie future de l'enfant et était porteuse de valeurs, notamment pour les jeunes filles provenant de la classe ouvrière. Elle se faisait dans l'optique où les qualités exigées de l'enfant en institution anticipaient celles qui lui seraient demandées plus tard, que ce soit dans le marché du travail ou dans le foyer familial ${ }^{39}$. Tout indique que c'est dans cette perspective qu'on entrevoyait l'éducation à l'Hospice Saint-Joseph.

À l'encontre des institutions d'enfermement, les contacts entre les parents et les enfants étaient privilégiés à l'Hospice Saint-Joseph. Ainsi, dans les Chroniques, nous avons constaté à quelques reprises que des parents ont pu assister à certains événements de la vie de leur enfant, comme à leur première communion ${ }^{40}$. En ce sens, comme le souligne Judith Ann Dulberger, dans son étude sur l'Albany Orphan Asylum, l'orphelinat n'agissait pas comme lieu de séparation pour briser la «ligne du paupérisme» ou le cycle de pauvreté, mais bien comme un refuge pour empêcher les familles d'éclater lors d'une situation précaire ${ }^{41}$.

Nous avons également pu observer ce maintien du lien entre les enfants et leur famille à travers les conditions de sortie des orphelines. Tout comme l'a constaté l'historienne Janice Harvey pour le cas de deux institutions protestantes pour enfants, il semblerait que les administratrices de l'Hospice Saint-Joseph, dans leur vision traditionnelle de la charité, n'aient pas privilégié le système de placement dans des familles d'accueil. En effet, les administratrices de l'Hospice Saint-Joseph auraient plutôt

36. Nicole Thivierge, Histoire de l'enseignement ménager-familial au Québec 1882-1970 (Québec, Institut québécois de recherche sur la culture, 1982), 50.

37. Le Diocèse de Montréal à la fin du dix-neuvième siècle (Montréal, Sénécal et Cie imprimeur-éditeurs, 1900), 186.

38. D. Lemieux, «La socialisation des filles dans la famille», loc. cit., 254.

39. Pascale Quincy-Lefebvre, Familles, institutions et déviances. Une histoire de l'enfance difficile: 1880-fin des années trente (Paris, Économica, coll. «Économies et sociétés contemporaines», 1997), 369.

40. A.S.G.M. L10 2H3, L’Hospice Saint-Joseph de Montréal, [copie dactylographiée], Chroniques manuscrites (Octobre 1892 à octobre 1912), 21 mai 1896, 91.

41. J.A.Dulberger, op. cit., 3. 
préféré garder les orphelines entre les murs de leur institution, en exigeant d'elles une contribution aux tâches d'entretien et de financement, avant de les remettre à leur famille naturelle. Ainsi, mis à part les quelques cas de placement en famille d'accueil (dans 5,2\% des cas), de décès (dans 4,7\% des cas) ou de désertion (dans $1 \%$ des cas), les jeunes filles ont normalement été remises à un membre de leur parenté lors de leur sortie (dans $81,7 \%$ des cas). Notons également que 23 d'entre elles (dans $1,6 \%$ des cas) sont entrées au Noviciat de la Communauté après leur séjour à l'institution.

Dans cette optique, l'Hospice Saint-Joseph remplaçait de façon temporaire les parents selon la logique protective proposée dans la classification de l'historien Timothy Hasci ${ }^{42}$. Conformément à cette catégorie la plus couramment attribuée aux institutions catholiques, les Sœurs exerçaient un contrôle qui se voulait protecteur sur les enfants en leur donnant une éducation axée sur la religion catholique et en privilégiant, quoique de façon encadrée, les contacts avec le monde extérieur. Voilà pourquoi les orphelines accueillies à l'Hospice retournaient généralement dans leur famille après un séjour d'une ou deux années.

La volonté manifeste de plusieurs parents d'y envoyer leur enfant pour qu'elle y soit logée, nourrie et formée, tout en maintenant avec elle un lien, indique à notre avis que l'Hospice Saint-Joseph n'a pas été qu'une institution disciplinaire, et qu'il a été dirigé par des Sours qui avaient leur propre conception des rapports qui devaient caractériser la vie à l'intérieur de l'institution.

Les Sours devaient veiller jour et nuit sur les orphelines. Le travail d'éducation qu'elles menaient auprès d'elles, en plus de l'entretien des lieux et des tâches ménagères, leur demandait du temps. À cela s'ajoutaient des conditions financières précaires qui ont poussé les administratrices à faire preuve d'imagination pour diversifier les sources de financement et assurer ainsi la survie de leur institution au fil des années. Bien que cela se soit fait vraisemblablement sans que les Sœurs ne le veuillent, les différentes stratégies de financement auxquelles elles ont eu recours ont eu tendance à les éloigner de leurs missions de charité et d'éducation. Ainsi, des contradictions de plus en plus insoutenables se sont posées à la fin du siècle entre ces missions et les nécessités reliées à leur survie financière. Nous verrons, par exemple, que les orphelines deviendront littéra-

42. Timothy Hasci, Second Home: Orphan Asylums and Poor Families in America (Cambridge, Harvard University Press, 1997), 1. 
lement des ouvrières à l'intérieur même de l'institution afin de lui assurer cette survie.

\section{LES RESSOURCES HUMAINES ET MATÉRIELLES: LA SURVIE DE L'INSTITUTION}

\section{Les ressources humaines}

La différence entre les sexes dans la société occidentale de la seconde moitié du XIX ${ }^{e}$ siècle était reliée à l'ordre patriarcal de la société qui confinait la femme à la sphère privée et laissait la sphère publique à l'homme. La main-d'œuvre féminine catholique œuvrait ainsi sous une structure bien particulière. Au-dessus d'elle se situait la direction conventuelle de l'autorité épiscopale qui unissait l'ensemble des communautés, l'ensemble des institutions ${ }^{43}$. Ce n'est qu'au-dessous de cette hiérarchie dominée par des hommes que les œuvres pouvaient être coordonnées par les Supérieures des communautés et leur Conseil, puis être exercées par les Sours. Dans ce contexte, les femmes participaient au domaine "public» de la charité, mais sous l'approbation des hommes qui supervisaient le système d'assistance ${ }^{44}$. Les religieuses bénéficiaient de cette plus grande latitude puisqu'elles accomplissaient, dans le système d'assistance, des tâches associées étroitement à la maternité.

\section{Le personnel religieux}

La division du travail a rendu la vie collective possible au sein des institutions catholiques. Chaque Sœur avait sa responsabilité spécifique que l'on qualifiait souvent "d'office ${ }^{45}$ ». À l'Hospice Saint-Joseph, les orphelines étaient séparées dans des salles de l'institution en fonction de leur âge. La Salle de l'Ange-Gardien, construite plus tardivement, accueillait les "petites orphelines», "afin de donner à celles-ci des soins plus tendres tels que le réclame ce jeune âge ${ }^{46} \ldots$.. La salle de l’Enfant-Jésus hébergeait «les moyennes orphelines ", alors que la salle Sainte-Marie, plus grande, recevait les «grandes orphelines» qui devaient travailler à «l'Ouvroir». Dans la pratique, on retrouvait généralement une Sœur pour chaque salle de l'Hospice, parfois accompagnée d'une employée engagée. Du côté de la

43. Micheline D’Allaire, «L'originalité de l'œuvre sociale des Congrégations religieuses de Montréal aux XIX et XX ${ }^{\mathrm{e}}$ siècles», S.C.H.E.C. Études d'histoire religieuse, 59 (1993): 38.

44. Janice Harvey, The Protestant Orphan Asylum and the Montreal Ladies Benevolent Society: A Case Study in Protestant Child Charity in Montreal, 1822-1900, thèse de doctorat (histoire), McGill University, 2001, 271.

45. Tania Martin, «Housing the Grey Nuns: Power, Religion, and Women in fin-de-siècle Montréal», dans Annmarie Adams et Sally McMurry, dir., Perspectives in Vernacular Architecture, 7 (1997): 218.

46. A.S.G.M. L10 2H3, L’Hospice Saint-Joseph de Montréal, [copie dactylographiée],Chroniques manuscrites (Octobre 1892 à octobre 1912), 4 novembre 1892, 30 janvier 1894, 38. 
Salle d'Asile, jamais plus de quatre Sœurs ne se sont occupées des 242 enfants qui y séjournaient quotidiennement en moyenne ${ }^{47}$. De telles conditions demandaient certes beaucoup de travail aux Sours qui, de plus, veillaient sur les orphelines la nuit.

Le nombre de Sœurs attitrées à l'Hospice Saint-Joseph a varié de huit à vingt entre les années 1874 et $1906^{48}$. Elles y ouvraient au sein en moyenne d'une douzaine d'offices qu'elles combinaient parfois : hospitalière des grandes orphelines, hospitalière des petites orphelines, "asilienne», enseignante à la classe des externes, enseignante au Jardin de l'Enfance, visitatrice des pauvres et des malades, préposée à la sacristie, au parloir ou au dispensaire, cuisinière, surveillante des réfectoires, aide à la pharmacie, économe ou musicienne ${ }^{49}$.

Il n'est pas difficile d'imaginer le rythme de travail effréné au quotidien. Quelques bribes des Chroniques de l'institution nous l'ont laissé entrevoir:

Sœur Alphonse succombe sous le poids de sa lourde charge d'hospitalière, aussi toujours presque seule ${ }^{50}$ !!! Sœur Valois nous est donnée pour aide. [...] elle sera chargée des malades, seconde à la pharmacie, maîtresse de classe chez nos ouvrières, puis gardienne au parloir, au besoin. Pauvre Sour! Elle en aura suffisamment, à coup sûr ${ }^{51}$.

L'état d'épuisement des Sœurs et leur besoin de repos forcé ont souvent été abordés dans les Chroniques. On y mentionne, par exemple, que la Supérieure de l'institution, en 1904, accablée de travail, "continue de se surmener, mais le médecin venu hier la force à prendre du repos ${ }^{52}$ ». «La fatigue a rendu notre chère Sour Hubert malade et aujourd'hui elle est obligée de se reposer [...]", ajoute-t-on en $1906^{53}$. La chroniqueuse nous fait également remarquer qu'à certains moments, l'épuisement est général: «[...] toutes les Sœurs sont épuisées et malades», disait-elle en février $1899^{54}$.

47. Micheline Dumont, «Des garderies au $19^{\mathrm{e}}$ siècle : les salles d'asile des sœurs Grises à Montréal», dans Micheline Dumont et Nadia Fahmy-Eid, dir., op. cit., 273.

48. Données compilées à partir de A.S.G.M., L10 E, 2, Hospice Saint-Joseph Montréal, Personnel religieux au 31 décembre, 1874 à 1906.

49. Informations tirées de A.S.G.M., L10 E, 2, Hospice Saint-Joseph Montréal, Personnel religieux au 31 décembre, 1874 à 1906.

50. A.S.G.M. L10 2H3, L’Hospice Saint-Joseph de Montréal, op. cit., $1^{\text {er }}$ mars 1907, 367.

51. Ibid., 27 août 1907, 390 .

52. Ibid., 16 décembre 1904, 271.

53. Ibid., 26 avril 1906, 330.

54. Ibid., 12 février $1899,170$. 
Sans surprise, l'Hospice connaissait un grand roulement de personnel ${ }^{55}$. Cela était en partie attribuable aux conditions de travail, mais également aux pratiques internes de la Communauté. Conformément aux exigences de cette dernière, les Sœurs faisaient chaque année une retraite annuelle lors de laquelle elles devaient se présenter à la Supérieure "pour lui rendre compte de l'emploi dont elle [était] chargée, et lui demander ses avis et ses conseils ${ }^{56}{ }^{\prime}$. C'est après cette retraite annuelle que les Sours, souvent, étaient changées d'office à l'intérieur de l'institution ou étaient, dans des cas plus extrêmes, envoyées dans une autre institution de la Communauté. Ces changements n'étaient pas sans déstabiliser les Sœurs qui avaient parfois peu de temps pour s'adapter à leurs nouvelles fonctions. En ce sens, nous avons pu lire dans les Chroniques, à quelques reprises, des réactions plutôt réfractaires à ces changements d'institution telles que: «Mes Sœurs retraitantes nous reviennent toutes converties [...] toutes ont échappé aux changements. Deo Gratias $^{57}$ !!» ou encore «[...] les départs ne sont pas propres à nous réjouir ${ }^{58}$ ".

\section{Le personnel laïque}

Chaque année, un nombre important de personnel non religieux venait compléter le travail des Sœurs dans l'institution. Ce personnel laïque, à gage ou non, occupait différents offices. Il devait soutenir les religieuses dans leur travail quotidien d'entretien de la cuisine, des réfectoires, de la lingerie, et de soins des enfants.

Les «femmes et filles», telles qu'on les nommait aux registres de l'Hospice, travaillaient sans recevoir de gages, le plus souvent pour de courtes durées. Elles étaient principalement des veuves, des femmes âgées et des filles «données» à l'institution ${ }^{59}$. Des hommes qualifiés d' "hommes pauvres», qui apparaissaient dans une proportion beaucoup plus faible aux

55. À cet effet, les registres du personnel religieux de l'institution nous ont permis de constater que, parmi les 180 Sœurs répertoriées entre les années 1872 et 1906, 65 sont restées dans l’institution pendant moins d'un an. Les 115 autres Sœurs ont, pour leur part, été attitrées à l'Hospice pour des durées de plus d'un an, pendant lesquelles elles se sont souvent promenées d'office en office.

56. BAnQ, MIC B524 13837 GEN, op. cit., 121.

57. A.S.G.M. L10 2H3, L’Hospice Saint-Joseph de Montréal, op. cit.,13 août 1906, 340.

58. Ibid., 7 septembre $1905,300$.

59. Les «filles données» entraient souvent dans l'institution en fournissant une somme en argent et en faisant la promesse d'y travailler, en échange de quoi la Supérieure s'engageait à ce que l'institution réponde à leurs besoins jusqu'à leur mort. Elles vivaient comme des religieuses, mais n'avaient pas prononcé leurs vœux. Elles ne devaient pas s'attendre à d'autres salaires que la nourriture quotidienne, un toit et la garantie qu’elles seraient assistées lorsqu'elles atteindraient un âge plus avancé. Cité dans Tania Martin, «Housing the Grey Nuns: Power, Religion, and Women in fin-de-siècle Montréal», dans Perspectives in Vernacular Architecture, loc. cit., 218. En plus de faire leur lit et leur "petit ménage» chaque jour, elles devaient participer à la fabrication des objets vendus pour assurer des revenus à l’institution. Bettina Bradbury, «Mourir chré- 
registres de l'Hospice, semblent avoir été le pendant masculin de ces "femmes et filles». Selon leur état physique, ils devaient effectuer certaines tâches ${ }^{60}$ en échange de nourriture et d'un lit. Il semblerait que ce personnel ait été évincé progressivement de l'institution en raison du fait qu'il générait peu de revenus, alors qu'il coûtait cher d'entretien et prenait de l'espace entre les murs de l'établissement, un espace nécessaire pour les orphelines et l'Ouvroir toujours de plus en plus imposant.

La diminution graduelle du nombre de ces pensionnaires a été compensée par l'embauche de femmes et d'hommes qui recevaient des gages pour leur travail. Ce personnel était composé majoritairement de femmes, mais quelques hommes s'y ajoutaient chaque année pour accomplir surtout les tâches de charretier et d'homme de cour. Ainsi, dix-huit femmes et deux hommes en moyenne par année travaillaient contre rémunération à l'Hospice. Ce personnel au fort roulement engendrait des dépenses qui représentaient environ une proportion de $5 \%$ à $10 \%$ des dépenses annuelles totales de l'institution. Les gages versés étaient dérisoires. Aux Chroniques, on dit d'un homme qui y est resté peu de temps: «Notre bon Joseph $[\ldots]$ nous a quittés hier à son grand regret. Il aime Saint-Joseph, mais les gages que nous pouvons lui donner ne lui conviennent pas ${ }^{61}$.» Il va sans dire que la durée de séjour des femmes embauchées, encore moins bien payées que les hommes comme Joseph, a rarement dépassé une année. En tout, sur les 76 cas d'hommes et de femmes à gages de l'échantillon, 58 personnes ont été au service de l'institution pendant moins d'un an, soit un peu plus de $75 \%$ d'entre elles.

Les Sœurs se sont heurtées à des limites importantes en termes de personnel, ce qui a poussé à leur épuisement. Le jour, elles devaient s'occuper d'une centaine d'orphelines et des quelques centaines d'enfants qui fréquentaient la Salle d'Asile en plus des tâches ménagères, de la gestion administrative et d'offrir différents services aux pauvres et aux malades. À ce travail constant, il fallait ajouter la charge de travail liée à la survie financière de l'institution. En 1907, on lisait dans les Chroniques: «que c'est triste d'avoir affaire aux personnes du monde pour vivre ${ }^{62}$ !» Cette phrase était fort révélatrice de la volonté d'autonomie qui animait les Sœurs et

tiennement: la vie et la mort dans les établissements catholiques pour les personnes âgées à Montréal au XIX siècle", Revue d'histoire de l'Amérique française, 46,1 (été 1992): 161.

60. B. Bradbury, «Mourir chrétiennement... », loc. cit., 161.

61. A.S.G.M. L10 2H3, L’Hospice Saint-Joseph de Montréal, [copie dactylographiée], Chroniques manuscrites (Octobre 1892 à octobre 1912), 16 mai 1899, 181.

62. Ibid.,22 janvier 1907, 363. 
de la lourdeur de la recherche incessante de fonds à laquelle elles étaient soumises.

\section{Les ressources matérielles}

Comme pour la plupart des institutions charitables, les administratrices de l'Hospice n'avaient pas le pouvoir de s'endetter, mais seulement celui d'ajuster les dépenses aux dons reçus et aux revenus générés. On comprend ainsi pourquoi les dépenses et les revenus de l'Hospice Saint-Joseph sont restés équilibrés au fil des années (Figure 1). Les administratrices ont d'abord reçu de l'aide financière de la Maison mère, puis elles ont rapidement multiplié leurs sources de revenus, comme le faisaient également la plupart des autres institutions charitables à l'époque ${ }^{63}$.

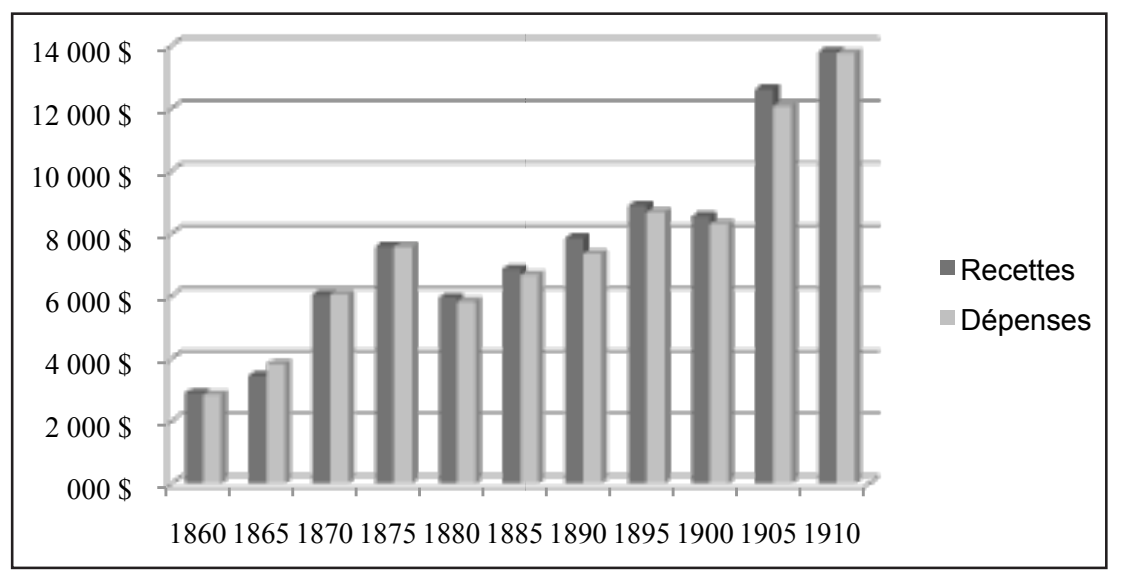

Figure I

Recettes et dépenses totales de l'Hospice Saint-Joseph (1860-1910)

Données compilées à partir de A.S.G.M., LI0 D3 I, Hospice Saint-Joseph de Montréal, comptes rendus à la Maison Mère, I854-I886 et de A.S.G.M., LIO D3 2, Hospice SaintJoseph de Montréal, comptes rendus, I886-1915.

La nourriture périssable constituait la principale dépense, avec une proportion de plus de $41 \%$ des dépenses totales. Venaient ensuite le chauffage, les marchandises sèches, l'eau, l'entretien des animaux, le matériel religieux, l'embauche de personnel, l'entretien du bâtiment, le matériel pour le bureau, la classe ou l'entretien ménager, les frais de déplacement et les assurances. 
Bien que les institutions charitables de la seconde moitié du XIX ${ }^{e}$ siècle aient touché une partie importante de la population, les pouvoirs publics les finançaient peu, sauf par les exemptions de taxes municipales ${ }^{64}$. Quant aux subventions provinciales, elles ne totalisaient pas plus de $10 \%$ des revenus des Sœurs Grises ${ }^{65}$. Les administratrices s'en remettaient donc davantage aux dons, parmi eux, ceux du Séminaire Saint-Sulpice et de la Banque d’Épargne. Au fil des années, les montants versés par ces deux entités furent toutefois de moins en moins suffisants.

Les Dames de la charité de l'élite montréalaise aidaient aussi grandement les administratrices de l'institution sur le plan financier. Elles faisaient les comptes rendus mensuels de la situation de l'institution en assemblée générale ${ }^{66}$, contribuaient par des sommes d'argent et organisaient des activités de financement auprès de la population. L'organisation d'événements au bénéfice de l'Hospice Saint-Joseph par ces Dames était une pratique courante. Nous avons constaté aux registres financiers que l'organisation de bazars, de loteries ou de soirées a permis d'amasser des montants qui ont représenté jusqu'à $15 \%$ de ses recettes annuelles totales au fil des années. Le 25 janvier 1893, par exemple, un souper-bénéfice donnait $500 \$$ à l'Hospice. Le 29 mars $1898^{67}$, un autre souper organisé par ces Dames rapportait à l'institution la somme de $1030 \$^{68}$, une somme qui représentait plus de $12 \%$ de ses revenus annuels, amassée en une seule soirée.

Les Sœurs comptaient également sur les dons de la population. Comme sœur Charlebois le mentionnait dans une lettre à $\mathrm{M}^{\text {gr }}$ Bourget en 1869: «[...] quand je ne puis payer un compte, je le fais porter par une enfant aux pieds de St Joseph et toujours il est payé sans faire souffrir ses débiteurs ${ }^{69}$ ». Afin d'obtenir des dons, il fallait continuellement démontrer l'utilité de l'institution aux bienfaiteurs et aux paroissiens et convaincre qu'elles soulageaient efficacement la misère et la pauvreté de la population $^{70}$. Ainsi, les dons étaient souvent octroyés à la suite de veilles et de prières faites à l'endroit de certains bienfaiteurs ou de leur famille, en guise

64. Y compris la taxe d'eau. Huguette Lapointe-Roy, op. cit.,135-138.

65. Ibid., 68.

66. Ibid., 92.

67. A.S.G.M. L10 2H3, L’Hospice Saint-Joseph de Montréal, op. cit., 25 janvier 1893, 10.

68. Ibid., 29 mai 1898, 142.

69. A.S.G.M., L10 C2 25, Sœur Charlebois [Document sans titre, lettre à $\mathrm{M}^{\mathrm{gr}}$ Ignace Bourget], 14 juillet 1869,1 . Souligné dans l'original.

70. Tania Martin, «La mouvance de la culture canadienne-française vue à travers l'évolution d'une institution catholique en région ", The Canadian Historical Review, 88,1 (mars 2007): 41-88. 
de remerciement à l'institution. En plus de ces aumônes et de ces legs, les Sours touchaient chaque année des recettes grâce aux troncs (des boîtes destinées à recevoir des aumônes dans les lieux religieux), aux quêtes ou aux locations de bancs à la chapelle de l'Hospice. Comme l'a démontré Louise Gagnon-Arguin pour l'Orphelinat Saint-Antoine au Saguenay, plusieurs institutions, et notamment les orphelinats, étaient sous la protection de mécènes ${ }^{71}$. À l'Hospice Saint-Joseph, ce sont le fondateur et ses descendants qui ont joué ce rôle. Le curé de la paroisse, fréquemment de passage dans l'institution, contribuait également au financement en faisant des dons lors de ses venues.

Nous l'avons vu, devant les besoins financiers croissants de la fin du $\mathrm{XIX}^{\mathrm{e}}$ siècle, les religieuses ont commencé à exiger des pensions aux parents qui désiraient placer leur jeune fille et aux femmes et filles qu'elles hébergeaient, ce qui entrait partiellement en contradiction avec leur mission charitable. Cette contribution financière que le parent, un membre de la famille élargie ou un bienfaiteur apportait à l'institution variait entre $1 \$$ et $6 \$$ par mois. En tout, en 1900 , l'Hospice recevait des pensions pour environ $40 \%$ des orphelines. Même en supposant que les pensions étaient d'une moyenne d'un peu plus de $3 \$$ par mois, et donc d'environ $40 \$$ par année, les revenus annuels associés à cette pratique ne pouvaient guère être de plus de 2000 \$, ce qui était loin de permettre de joindre les deux bouts.

Les administratrices de l'Hospice Saint-Joseph détenaient également des propriétés foncières à Montréal et à Châteauguay, qui ont contribué, par leur vente ou leur location, à la survie financière. Les revenus étaient principalement tirés de la location d'espaces dans certains de leurs édifices $^{72}$. Entre 1865 et 1900 , ces revenus immobiliers étaient modestes et représentaient environ $5 \%$ des revenus totaux de l'institution, mais entre les années 1905 et 1910, ces revenus ont connu une hausse importante et ont ainsi occupé une place prépondérante parmi les sources de revenus. En 1911, alors que l'Hospice est passé au statut d'école ménagère, le montant que rapportaient les biens immobiliers s'élevait à plus de 57\% de ses revenus totaux ${ }^{73}$.

71. Louise Gagnon-Arguin, «L'orphelinat St-Antoine et ses bienfaiteurs », Saguenayensia, 26,3 (juilletseptembre 1984): 92.

72. Tout comme c'était le cas de plusieurs institutions charitables. J.-M. Fecteau et J. Harvey, «Le réseau de régulation sociale montréalais", op. cit.

73. A.S.G.M., L10 RAPPORTS 1 67, Personnel et Euvres de la maison de l'Hospice Saint-Joseph, Montréal, $1^{\text {er }}$ septembre 1911 au $1^{\text {er }}$ septembre 1912. 
La fermeture de plusieurs services démontre que l'Hospice a abandonné progressivement sa mission charitable au tournant du $\mathrm{XX}^{\mathrm{e}}$ siècle. En 1902, par exemple, la Salle d'Asile Saint-Joseph a été fermée ${ }^{74}$. Selon les dires des Sours, "la vie des enfants [était] trop exposée, à cause du site commercial» qui s'était développé autour de l'Hospice ${ }^{75}$. Dans le quartier, selon sœur Gaudry, les rues étaient devenues plus commerciales, entourées d'hôtels, de restaurants et de véhicules qui mettaient en danger la vie des enfants et éloignaient les habitants du faubourg. Cela étant dit, il est indéniable que des raisons financières ont également poussé les Sœurs à fermer ce service. En effet, un an après sa fermeture, les administratrices louaient aux Sulpiciens ces locaux "afin de créer des revenus pour venir en aide à l'Hospice ${ }^{76}$ ». Pour les mêmes raisons, en juin 1904, le service du Jardin de l'Enfance était supprimé et sa bâtisse devenait un magasin ${ }^{77}$. La chroniqueuse écrivait: "Aujourd'hui on commençait le déménagement du Jardin de l'Enfance, cette œuvre non plus que celle des salles d'Asile n'existera dans le quartier. La Maison mère prend possession de cette bâtisse pour la convertir en "magasin"78." Puis, en août 1904, c'est le dispensaire qui fermait ses portes ${ }^{79}$. En raison d'une nouvelle division des paroisses, les Sulpiciens avaient cessé les versements annuels qui y étaient destinés. Les Sœurs, par conséquent, se déclaraient : «dans l’impossibilité de continuer cette œuvre [...] au grand regret des médecins ${ }^{80}$ ».

Finalement et surtout, les administratrices de l'Hospice accordaient une place primordiale aux ouvrages que les Sours réalisaient en compagnie des orphelines dans l'Ouvroir de l'institution. "Cette maison, on le sait, ne subsiste que d'aumônes et des ouvrages faits par les grandes orphelines qui sont admirables de courage, de bonne volonté et surtout de dexté-

74. Certaines raisons, autres que financières, expliquent également la fermeture de la Salle d'Asile Saint-Joseph. Tout comme les autres Salles d'Asile à Montréal, sa popularité va décroître à la fin du XIX siècle et elle sera appelée à disparaître au début du $\mathrm{XX}^{\mathrm{e}}$ siècle. Voir à ce sujet Micheline Dumont, "Des garderies... ", loc. cit., 281-284; Micheline Lalonde-Graton, Des salles d'asile aux centres de la petite enfance: la petite histoire des services de garde au Québec (Québec, Les Presses de l’Université du Québec, 2002), 9-10; et Denyse Baillargeon, «Les politiques familiales au Québec. Une perspective historique», Lien Social et Politiques- RIAC, 36 (automne 1996): 23.

75. A.S.G.M., L10 CI 14, Sœur Jean de la Croix, Prise de possession de l'Hospice St Joseph, non daté, 6.

76. A.S.G.M., L10 F6 60, Sœur McKenna, dépositaire [Document sans titre], 25 juin 1903, 1.

77. A.S.G.M., L10 CI 14, Sœur Jean de la Croix, op. cit., 6.

78. A.S.G.M. L10 2H3, L’Hospice Saint-Joseph de Montréal, [copie dactylographiée], Chroniques manuscrites (Octobre 1892 à octobre 1912),13 juin 1904, 249.

79. A.S.G.M., L10 CI 14, Sœur Jean de la Croix, op. cit., 6.

80. Le dispensaire recevait des Sulpiciens en moyenne 150 \$ par année. A.S.G.M. L10 2H3, L’Hospice Saint-Joseph de Montréal [copie dactylographiée] Chroniques manuscrites (Octobre 1892 à octobre 1912), 5 août 1904, 254. 
rité $^{81} . . . »$, disait la chroniqueuse de l'Hospice au sujet de cette source de revenus qui a souvent dépassé $30 \%$ de l'ensemble des recettes annuelles. À leur sommet, nous avons pu observer que ces revenus ont représenté plus de $65 \%$ des revenus totaux de l'institution. Ces «industries» comportaient principalement des travaux de couture et, à de plus faibles proportions, la conception de certains objets comme des cierges et des tricots.

Avant 1856, les travaux de couture étaient exécutés à la main par les Sours et les orphelines, mais en 1856 le sulpicien Victor Rousselot a fait don d'une machine à coudre à l'Hospice. Les Sours voyaient dans cet instrument un nouveau moyen d'accroître leurs revenus et de demeurer «compétitives» dans le domaine de la couture. À notre avis, cette première machine à coudre a constitué la prémisse de travaux qui, plusieurs années plus tard, allaient faire de l'Hospice une institution qui se rapprocherait drôlement d'une manufacture. Un pas de plus était franchi en 1906, alors que 20 jeunes filles étaient employées aux machines à coudre à l'électricité qui étaient prêtées "gratis » à l'institution par le propriétaire d'une manufacture. Ce propriétaire, disait la chroniqueuse, «s'engage à nous fournir autant d'ouvrage que nous pourrons en faire ${ }^{82} »$. Finalement, au tournant des années 1910, l'Hospice était littéralement au service d'un propriétaire de manufacture qui s'engageait à lui laisser les machines aussi longtemps que les orphelines travailleraient pour lui. Les administratrices de l'institution s'en réjouissaient puisque cette entente leur "procur[ait] de l'ouvrage ${ }^{83}$ " et, par le fait même, des ressources financières.

Notons qu'en raison des revenus qu'il générait, l'Ouvroir a occupé de plus en plus de place dans le bâtiment au fil des années, empiétant constamment sur les espaces consacrés aux orphelines. En 1894, par exemple, on jetait les fondations de la galerie couverte dans le but de l'agrandir, car il était devenu trop étroit pour le nombre d'ouvrières et la quantité de travaux qu'on y réalisait ${ }^{84}$. En 1908, c'est le dortoir que l'on réaménageait afin d'en employer une partie pour les travaux de couture ${ }^{85}$. Le travail de couture, qui s'y est industrialisé au fil des années, nous porte à croire que le besoin incessant de fonds a progressivement éloigné l'Hospice SaintJoseph de sa mission charitable en faisant littéralement travailler les orphelines pour assurer sa subsistance. Ainsi, tout comme c'était le cas

81. Ibid., 17 septembre 1893, 29.

82. Ibid., 8 janvier 1906, 318.

83. Ibid., 26 février 1909, 433.

84. Ibid., 30 mai 1894, 46.

85. Ibid., 27 mars 1908, 415 . 
dans d'autres institutions ${ }^{86}$, c'est en dépit de leur mission de protection à l'enfance que les Sœurs ont dû soumettre les orphelines à un travail constant, pour répondre à des besoins matériels essentiels.

À bout de souffle, au tournant du $\mathrm{XX}^{\mathrm{e}}$ siècle, le personnel restreint et en mouvement et la situation financière précaire ont contraint l'Hospice Saint-Joseph à délaisser progressivement sa mission de charité. La mission charitable, continuellement mise à l'épreuve, a finalement été supplantée par un projet institutionnel qui rendait la situation financière nettement moins précaire. En 1911, les administratrices de l'Hospice convertissaient l'Ouvroir, leur veau d'or, en une école ménagère. Elles délaissaient ainsi la politique "d'open door» de leur Communauté pour laisser toute la place à l'éducation ménagère de jeunes filles mieux nanties ${ }^{87}$. Elles justifiaient leur nouvelle mission par l'attribution du rôle plus strictement défini de la femme qui prévalait au début du $\mathrm{XX}^{\mathrm{e}}$ siècle, une situation qui demandait une formation ménagère plus poussée pour les jeunes filles

[...] ces jeunes orphelines dont on a fait souvent dans le passé de bonnes mères de famille il faut songer à les mieux armer encore pour le ménage, la couture, les soins domestiques... L'ouvroir est devenu une École Ménagère sur l'organisation d'une maison, sur l'alimentation, le vêtement, les soins à donner, la cuisine à faire, la propreté à conserver, tout enseignés aux chères fillettes ${ }^{88}$.

Ce changement de mission n'a certainement pas été étranger au penchant de l'Église catholique pour les écoles ménagères. En effet, au début du $\mathrm{XX}^{\mathrm{e}}$ siècle, le comité catholique du conseil de l'Instruction publique recommandait la création de plusieurs écoles ménagères dans la province de Québec. Ce comité catholique, composé à $50 \%$ d’Évêques, redistribuait lui-même l'aide financière de l'État aux écoles ménagères et leur fournissait un soutien financier, notamment pour la transformation des institutions en écoles ménagères ${ }^{89}$. De plus, ces écoles relevaient souvent

86. Dale Gilbert a notamment constaté ce phénomène dans son étude de l’Hospice Saint-Charles de Québec, une école de réforme et d'industrie des Sœurs du Bon-Pasteur qui agissait également à titre d'orphelinat dans la ville de Québec entre les années 1870 et 1950. Dale Gilbert, "Dynamique de l'institutionnalisation de l'enfance délinquante et en besoin de protection: le cas des écoles de réforme et d'industrie de l'Hospice Saint-Charles de Québec, 1870-1950", mémoire de maîtrise (histoire), Université Laval, 2006, 89.

87. Bien que les Sœurs aient accueilli une clientèle constituée presque exclusivement d'orphelines en âge de suivre les cours ménagers après 1911, notons qu'elles ont tout de même poursuivi leur œuvre auprès des pauvres dans le cadre de visites à domicile et de repas donnés en service externe par la suite. A.S.G.M., L10 E1 2, Asile Saint-Joseph (adjacent à l'Hospice) et Hospice. Rapport (1863-1935).

88. A.S.G.M., L10 C19 [Document sans titre, notes manuscrites sur l'Hospice], non daté, 1.

89. Nicole Thivierge, Histoire de l'enseignement ménager-familial au Québec 1882-1970 (Québec, Institut québécois de recherche sur la culture, 1982), 149. 
de l'initiative d'un curé ou d'un évêque qui demandait toujours une aide gouvernementale pour les faire fonctionner. Grâce à cette aide financière, les Sœurs de l'Hospice Saint-Joseph pouvaient enfin poursuivre leur œuvre dans de meilleures conditions, bien qu'avec une clientèle différente. Ainsi, à partir de 1911, 70 jeunes filles de 14 ans et plus ont été accueillies à l'École ménagère Saint-Joseph chaque année ${ }^{90}$.

L'aspect financier a-t-il à lui seul engendré la transformation de l'Hospice en une école ménagère? Bien que de nouvelles valeurs insistant sur l'importance du foyer familial comme lieu d'éducation pour les enfants aient commencé à se répandre au tournant du $\mathrm{XX}^{\mathrm{e}}$ siècle ${ }^{91}$, nous croyons que c'est davantage le facteur économique qui a mené à la fin de sa mission charitable. Le courant idéologique qui visait à ne pas séparer les enfants de leurs parents pour des raisons de pauvreté s'est installé bien lentement au Québec et n'a vraisemblablement eu que peu d'impact sur la fermeture de l'Hospice Saint-Joseph. Certes, des sujets tels que l'aide aux familles pauvres et la désinstitutionalisation des enfants ${ }^{92}$ ont été abordés lors de l'Exposition pour le Bien-être de l'enfance, tenue à Montréal en 1912, mais ces idées étaient toujours contestées, ce qui explique qu'il faudra attendre le milieu des années 1930 pour que cela se traduise, par exemple, par un programme de pensions aux mères nécessiteuses.

\section{CONCLUSION}

À travers cette histoire de l'Hospice Saint-Joseph que nous avons proposée, nous avons pu constater que la volonté charitable de protection de l'enfance des Sœurs s'est heurtée au débordement de clientèle ainsi qu'au manque de personnel et au sous-financement chronique. Le système d'assistance catholique laissait une large part d'autonomie administrative aux Sœurs, mais ces dernières avaient toutefois peu de ressources pour agir.

L'Hospice Saint-Joseph n'a pas été un lieu d'enfermement destiné à briser "la ligne du paupérisme». Comme c'était le cas dans plusieurs orphelinats, tout semble indiquer que les Sœurs de l'Hospice ne visaient pas à «séparer» les familles comme le proclamaient les réformateurs du courant «anti-institutionnel». Elles cherchaient plutôt à maintenir des contacts avec les familles et n'hésitaient pas à retourner les enfants à leurs

90. A.S.G.M., L10 C2 15 [Document manuscrit sans titre], non daté, 1.

91. Notamment aux États-Unis lors de la White House Conference on Children en 1909.

92. J.-M. Fecteau, La liberté du pauvre: crime et pauvreté au XIXe siècle québécois, op. cit., 199. 
parents dès qu'ils étaient en mesure de les recevoir. L'Hospice a donc été un refuge où des parents pouvaient envoyer leur enfant à court ou à long terme. Ainsi, il s'est montré ouvert tant aux familles en crise du cycle de la vie ou en crise financière qu'envers celles qui recherchaient un lieu de formation pour leur enfant. À tous ceux qui y étaient admis, il offrait la nourriture, un toit et une éducation fondée sur la religion catholique.

Avant la Loi sur l'assistance publique de 1921, l'État québécois a été très peu présent dans le domaine de l'assistance, et cela même en le comparant avec d'autres États libéraux. C'est donc à la société civile qu'est revenue la tâche de secourir les indigents. Un peu partout en Amérique du Nord, c'est l'élite bourgeoise qui a joué un rôle essentiel dans le développement des systèmes d'assistance, promouvant ainsi sa propre conception de la pauvreté et de la charité. Au Québec, toutefois, la bourgeoisie canadiennefrançaise a joué un rôle marginal et essentiellement financier face aux institutions catholiques administrées par le clergé et gérées par les Sours.

Le placement des enfants en institution, tout comme leur "placement» à l'école ou sur le marché du travail, figurait parmi les stratégies de divers types de familles dans la seconde moitié du XIX ${ }^{\mathrm{e}}$ siècle. L'analyse d'un échantillon de cas a, en effet, révélé que différents types d'orphelines ont fréquenté l'Hospice Saint-Joseph. Peu importe leur provenance, toutes devaient apprendre la couture, une compétence qui semblait condenser toutes les valeurs que l'on attendait d'une future mère. Bien sûr, la couture assurait également un revenu à l'Hospice.

Les Sœurs se sont heurtées à des limites importantes en termes de personnel. De surcroît, les normes de la Communauté, l'épuisement et le débordement des religieuses engendraient un mouvement de personnel important. Cette instabilité touchait aussi le personnel laïque, principalement constitués de femmes "données» et de femmes à salaire, qui souvent, quittaient l'institution faute d'obtenir un salaire décent.

Alors que l'Hospice ne pouvait s'endetter, les administratrices ont appris rapidement à diversifier les sources de financement qui allaient assurer la survie de leur institution. Les travaux de couture qu'elles réalisaient conjointement avec les orphelines et les Dames de la Charité leur rapportaient des profits importants, allant jusqu'à $65 \%$ de leurs revenus totaux. Au fil des années, les Sœurs ont d'ailleurs appris à se battre pour demeurer compétitives sur le marché de la consommation, notamment en agrandissant à plusieurs reprises l'Ouvroir et en négociant avec des propriétaires de manufactures pour leur soutirer des contrats. À la fin du XIX ${ }^{\mathrm{e}}$ siècle, l'Hospice a commencé à exiger de plus en plus fréquemment 
des pensions aux parents qui désiraient y placer leur jeune fille, ce qui entrait partiellement en contradiction avec la mission charitable de l'institution. À cela s'ajoutaient également la vente et la location de biens immobiliers, à partir du début du $\mathrm{XX}^{\mathrm{e}}$ siècle.

Essoufflées par les problèmes financiers, les religieuses ont progressivement délaissé la mission première de leur institution pour mettre fin à une expérience charitable de plus de cinquante années. Nous croyons que la fermeture de l'Hospice est un exemple de l'épuisement du réseau d'assistance catholique, tout comme ce fut le cas pour bien d'autres institutions d'assistance catholique fondées à partir du milieu du XIX ${ }^{e}$ siècle. C'est d'ailleurs pour cette raison que le gouvernement libéral a fait adopter la Loi sur l'assistance publique de 1921, qui visait à soutenir les institutions, notamment celles consacrées à l'enfance. Cette loi reconnaissait, un quart de siècle trop tard, tout le travail accompli par les Sœurs. 